Revista Arbitrada Interdisciplinaria KOINONIA

Año V. Vol V. №10. Julio - Diciembre 2020

Hecho el depósito de Ley: FA2016000010 ISSN: 2542-3088

FUNDACIÓN KOINONIA (F.K). Santa Ana de Coro. Venezuela.

Carlos Francisco López-Intriago; Juan Carlos Erazo-Álvarez; Cecilia Ivonne Narváez-Zurita; Verónica Paulina Moreno

http://dx.doi.org/10.35381/r.k.v5i10.701

\title{
Gestión financiera basada en la creación de valor para el sector microempresarial de servicios
}

\section{Financial management based on the creation of value for the services micro- business sector}

\author{
Carlos Francisco López-Intriago \\ clopez@psg.ucacue.edu.ec \\ Universidad Católica de Cuenca, Cuenca \\ Ecuador \\ https://orcid.org/0000-0003-3967-7580 \\ Juan Carlos Erazo-Álvarez \\ jcerazo@ucacue.edu.ec \\ Universidad Católica de Cuenca, Cuenca \\ Ecuador \\ https://orcid.org/0000-0001-6480-2270 \\ Cecilia Ivonne Narváez-Zurita \\ inarvaez@ucacue.edu.ec \\ Universidad Católica de Cuenca, Cuenca \\ Ecuador \\ https://orcid.org/0000-0002-7437-9880 \\ Verónica Paulina Moreno \\ veronica.moreno@ucacue.edu.ec \\ Universidad Católica de Cuenca, Cuenca \\ Ecuador \\ https://orcid.org/0000-0003-1517-6124
}

Recibido: 25 de marzo de 2020

Revisado: 05 de abril de 2020

Aprobado: 20 de abril de 2020

Publicado: 19 de mayo de 2020

\section{RESUMEN}

La investigación tuvo por objetivo diseñar un modelo de gestión financiera para el mejoramiento de la rentabilidad en la residencia estudiantil Nia de la Ciudad de Cuenca, con ello tratar de lograr un mejor posicionamiento en el mercado de las residencias 
estudiantiles de la ciudad y en la mente de los estudiantes universitarios. El diseño de la investigación fue de tipo no experimental, analizando las variables involucradas: gestión financiera basada en la creación de valor. La investigación fue de tipo mixta, con enfoque cuantitativo. La investigación concluye que el modelo de gestión financiera optimiza la creación de valor en la Residencia Estudiantil NIA; además, con los procedimientos respectivos y el tratamiento oportuno se hace posible que los objetivos de rentabilidad se vean alcanzados.

Descriptores: Desarrollo económico y social; educación y desarrollo; planificación del desarrollo; economía de la educación. (Palabras tomadas del Tesauro UNESCO).

\begin{abstract}
The objective of the research was to design a financial management model to improve profitability in the Nia student residence in Cuenca city, with the purpose of trying to achieve a better position in the market for city student residences and in the mind of university students. The research design was non-experimental; so, it was focus on the analysis of the variables involved: financial management based on value creation. The research was mixed, with a quantitative approach. The research concludes that the financial management model optimizes the creation of value in the NIA Student Residence; besides, with the respective procedures and the timely treatment, the profitability objectives could be achieved.
\end{abstract}

Descriptors: Economic and social development; education and development; development planning; economics of education. (Words taken from the UNESCO Thesaurus).

\title{
INTRODUCCIÓN
}

En Ecuador, en estas dos últimas décadas ha cambiado su sistema educativo. Se han realizado reformas en la educación superior en busca de universidades de altos estándares de calidad en todas las ciudades del país, incrementando así nuevas oportunidades para los jóvenes bachilleres de todo el país, esto desemboca en la creación de residencias estudiantiles. En ciudades como Quito y Guayaquil el mercado de residencias estudiantiles está más consolidado, en Cuenca se encuentra en una etapa de temprano desarrollo. Se destacan estas tres ciudades pues es donde se ubican las principales universidades del país. 
En la provincia del Azuay y de las provincias vecinas como lo son Cañar, El Oro, Loja, Morona Santiago y Zamora Chinchipe, llegan un gran porcentaje de estudiantes los cuales se radican en la ciudad de Cuenca provocando una alta demanda de alojamiento. La Residencia Estudiantil NIA, es una pequeña organización dedicada al alojamiento de estudiantes universitarios, ubicado en la ciudad de Cuenca perteneciente a la provincia del Azuay. Creada en 1999 con actividad comercial el alquiler de habitaciones y departamentos para estudiantes universitarios. Cuenta con 2 colaboradores, encargados de las áreas administrativa, operativa y bodega.

La residencia estudiantil NIA desde sus inicios brinda hospedaje a los estudiantes, un lugar donde se reúnen personas de diferentes géneros y costumbres, y se acogen a normas diseñadas para facilitar la convivencia y seguridad, un lugar donde pueden comer, dormir, estudiar, recibir servicios de limpieza.

El problema se enfoca en el bajo rendimiento financiero que tiene la residencia estudiantil NIA, ocasionando una débil rentabilidad ante el mercado residencial en momentos de alto flujo estudiantil; además, existe ausencia de un modelo de gestión financiero que permita un mayor control del capital y rentabilidad han ocasionado que la organización se detenga en cuanto a innovación de servicios, confort en sus habitaciones y mejoramiento de posicionamiento en el mercado; por ello, existen variables importantes que forman parte del objeto de estudio tales como capital circulante, gestión financiera, creación de valor y rentabilidad, mismas que permiten desarrollar el modelo ideal para obtener rentabilidad en la empresa.

La desventaja es la falta de un instrumento útil para la mejora de la rentabilidad, un sistema de gestión diseñado para responder a las necesidades de la actividad de hospedaje a estudiantes y permitiendo a la administración obtener mayor flujo de capital. Por el escenario antes expuesto, lo que se busca es diseñar un modelo de gestión financiera basada en la optimización de capital circulante y creación de valor que propicie el mejoramiento de la rentabilidad de la residencia estudiantil NIA de la ciudad de Cuenca. 
En esta perspectiva, el fundamentar teóricamente el criterio de autores nacionales e internacionales los modelos de gestión financiera, creación de valor y rentabilidad en las organizaciones; diagnosticar la situación actual de las residencias estudiantiles de la ciudad de Cuenca; para finalmente elaborar los componentes del modelo de gestión financiera para optimizar la creación de valor de la residencia estudiantil Nia de la ciudad de Cuenca.

\section{Referencial teórico}

\section{Modelo de gestión financiera, maneras de generar capital en organizaciones pequeñas}

Uno de los principales factores en la economía de una organización es el sistema monetario, dinero, en efecto es empleado para acelerar las transacciones mercantiles o comerciales como medio de pago para la obtención de un bien o servicio, según nuestra necesidad. Para llevar un registro de las operaciones comerciales y financieras, se emplea la contabilidad, con el objeto de efectuar la inspección de movimientos operativos y la correcta funcionalidad de la organización; mediante el control de transacciones, planificación de destrezas y operaciones futuras (Aibar-Ortiz, 2014). Las funciones principales de la moneda dentro de la organización son consideradas a continuación:

1. Medio de pago: forma parte de la sociedad en un estado y se extiende al intercambio de valores, bienes o servicios por el que se paga un valor determinado (Ramírez-Solano, 2001).

2. Unidad de cuenta: sirve de medio para desarrollar la contabilidad, convirtiéndose en un instrumento imprescindible para la organización (García-Gonzáles \& Boria-Reverter, 2006).

3. Depósito de valor: el dinero como tal es de facil manejo e intercambio, en efecto se puede reubicar el poder adquisitivo de un etapa a otra; por ejemplo, el par de 
divisas Euro/Dólar en estos tiempos de Covid19 hacen que su fuerza en el mercado bursatil varie de un instante a otro (Vargas-Sánchez, 2006).

4. Precio: Es el importe de riqueza, servicios y factores de la productividad expresados en términos del capital (Ortíz, 2003).

Por otro lado, (Parejo-Gámir; Calvo-Bernardino, Rodríguez-Sáiz, Cuervo-García \& Alcalde-Gutiérrez, 2016), mencionan que el dinero para su uso necesita una herramienta que denote las actividades financieras para la organización dentro de un periodo estipulado y que determine el grado de liquidez, por medio del flujo de caja se obtiene un estudio de la oferta y la demanda.

Para (Border \& Houpt, 2017), el mercado financiero es considerado una estrategia de intercambio de valores determinado por los costos que generan y las utilidaes recibidas durante sus transacciones; en efecto la tecnología y los sistemas empleados para el desarrollo de la misma han permitido que las negociaciones se realicen sin necesidad de la presencia de los accionistas de la organización.

\section{La gestión financiera como área clave de las organizaciones}

Las empresas pequeñas del país en estos tiempos para poder mantenerse y pasar el umbral necesitan un modelo de gestión financiera que permita por medio de la creación de valor la optimización del capital circulante y de esta manera mejorar la rentabilidad de la organización. Es fundamental analizar cada uno de estos componentes con el aporte de varios autores, que han publicado sus trabajos de relevancia.

La gestión financiera es el área clave y su principal objetivo es generar mayor valor o añadir riqueza a la organización. Esta función exige de sus ejecutivos habilidades especiales para las decisiones de inversión y financiamiento en ambientes de riesgo e incertidumbre. La gestión financiera involucra la utilización de instrumentos y técnicas mercantiles en donde el representante de este departamento deba incluir en su visión estratégica para lograr maximizar los recursos de la organización (Gonzáles-Urbina, 2016). 
Carlos Francisco López-Intriago; Juan Carlos Erazo-Álvarez; Cecilia Ivonne Narváez-Zurita; Verónica Paulina Moreno

Según (Córdoba-Padilla, 2012) uno de los ejecutores de la gestión financiera es el administrador financiero el cual cumple con un papel importante en la empresa, es la persona que debe planificar la adquisición y el uso de fondos mediante la optimización de recursos con el fin de que maximice el valor de la organización; el administrador de finanzas debe cumplir con las siguientes actividades:

1. Planeación y predicciones: son considerados como una interacción entre ejecutivos quienes serán los encargados de analizar las diferentes instancias de seguridad durante las transacciones comerciales que se realicen a favor de la organización.

2. Medidas adecuadas para financiación e inversión: consiste en seleccionar los tipos de inversiones a realizar para incrementar los clientes y los recursos.

3. Control: es la interacción entre el administrador y los ejecutivos de la empresa para la acción de operaciones eficientes para que generen rentabilidad y prestigio organizacional.

4. Interacción con los mercados de capital: es la relación entre la organización y los entes financieros.

Seguidamente, respecto a la gestión financiera los siguientes autores indican lo siguiente:

\section{Tabla 1}

Conceptos de la Gestión Financiera

Fuente

Conceptos de la Gestión Financiera

(Pérez-Carballo, 2015) "La misión general de la gestión financiera en la empresa de apoyar y financiar su crecimiento rentable, controlando su riesgo, comprende la formulación de objetivos, denominando como objetivo no un deseo sino un compromiso para alcanzarlo" (p.38).

Santiago-Chávez \& "La concepción moderna de la gestión financiera 
Revista Arbitrada Interdisciplinaria KOINONIA

Año V. Vol V. №10. Julio - Diciembre 2020

Hecho el depósito de Ley: FA2016000010

ISSN: 2542-3088

FUNDACIÓN KOINONIA (F.K). Santa Ana de Coro. Venezuela.

Carlos Francisco López-Intriago; Juan Carlos Erazo-Álvarez; Cecilia Ivonne Narváez-Zurita; Verónica Paulina Moreno

Gamboa-Salinas, empresarial, con su tratamiento conjunto e 2017)

(Terrazas-Pastor, "La Gestión Financiera es la actividad que se realiza en 2009) una organización y que se encarga de planificar, organizar, dirigir, controlar, monitorear y coordinar todo el manejo de los recursos financieros con el fin de generar mayores beneficios y/o resultados" ( $p .57)$.

Para (García-Gonzáles \& Boria-Reverter, 2005), la función de la gestión financiera a traves del administrador financiero es la de decidir, planificar, organizar, administrar y vigilar; y de esta manera conseguir el éxito de la organización.

Para la determinación del capital circulante; (Rojo, 2007) hace un análisis considerando la fracción del activo, siendo la operación matemática la resta del activo y pasivo corriente que se registran a un periodo contable. Cuando el resultado es positivo indica que exite una cantidad del activo que evidencia un capital propio para el negocio, ocurre lo contrario cuando la cantidad del pasivo es mayor y similar a los activos indica que se encuentra financiando los activos que generalmente suelen ser los activos no corrientes, con recursos fijos, concibiendo estos como la adición del patrimonio neto más el pasivo que da como resultado el capital del negocio.

Para (Milla, 2011) las decisiones financieras deben estar encaminadas a generar ingresos suficientes a fin de superar los costos operativos y generar el rendimiento deseado por la organización. Precisamente una de las características más relevantes con la relación al estudio de creación de valor es la de identificar las medidas desde una perspectiva interna hasta llegar a una perspectiva externa que contribuyan en el 
perfeccionamiento de la gestión empresarial. Bajo este criterio expuesto la asignación de valor en la organización es un concepto que se encuentra estrechamente relacionada con la estrategia que desarrollan las organizaciones.

Operando la organización en el mercado de bienes y capital, podrá determinar los beneficios que producen actividades comerciales o de servicios que ofrece y evaluar si los recursos económicos están disponibles con relación a sus costos por uso. Finalmente, deberá asignar eficientemente los fondos considerando las decisiones y acciones consecuentes y la etapa de retroalimentación. Esta función exige de sus ejecutivos habilidades especiales para las decisiones de inversión y financiamiento en ambientes de riesgo e incertidumbre. La gestión financiera involucra la utilización de instrumentos y técnicas mercantiles en donde el representante de este departamento deba incluir su visión estratégica (Murillo-Vilela, Erazo-Álvarez, Quevedo-Vázquez, \& Narváez Zurita, 2019). Dentro de esta perspectiva, vinculando al concepto de gestión financiera y residencias estudiantiles, se continua con el estudio de la creación de valor y de la rentabilidad.

\section{La creación de valor como resultado de la gestión financiera}

En este orden, (Prahalad \& Ramaswamy, 2004), consideran que la etapa a través del cual el comprador y el negocio se encuentran implicados en la generación conjunta de valor es la co-creación de valor la cual se convierte en la única imagen para el comprador; siendo para la organización un acto sostenible; el consumidor ha pasado de ser pasivo a estar activo y comprometido, de hecho el interes por adquirir un producto 0 servicio va acompañado de las experiencias vividas por el consumidor. Con la finalidad de garantizar las actividades operativas es importante que la organización posea recursos en stock que le permiten cumplir con la rotación normal de sus movimientos. Existen dos maneras que se pueden utilizar con el propósito de que los activos corrientes se mantengan y permitan la rotación operativa del negocio, estas son: 
Carlos Francisco López-Intriago; Juan Carlos Erazo-Álvarez; Cecilia Ivonne Narváez-Zurita; Verónica Paulina Moreno

1. Mediante la financiación de terceros, es decir, el crédito directo con los proveedores de las mercaderías o insumos, cuyo plazo tiene que estar comprendido entre 30, 45 y 90 días plazo.

2. Mediante uso de capital propio o dinero financiado a largo plazo mediante una entidad bancaria.

Los ciclos contables intermedios son similares para activo y pasivo circulante, además se encuentran establecido en base a las circunstancias económicas y financieras que deberan ser aprovechadas de acuerdo a las actividades operacionales que desarrolla la organización, como también otros factores administrativos, de manejo de recursos y desarrollo de las operaciones donde intervienen factores relacionados con:

1. La adquisición de elementos utilizados en la explotación comercial, programando fechas, pagos, convenios de descuentos.

2. La gestión de abastecimientos, la cual esta ligada con fechas específicas para las adquisiciones de los productos y manejo de stock.

3. La etapa productiva, comprende la estabilidad media que se considera en la selección de los tipo de productos en base a los gustos y preferencias de los clientes.

4. El control de inventarios sobre productos terminados, el cual consiste en la capacidad de almacenamiento, seguimiento de stock.

5. El cumplimiento de ventas, abarca los medios que se utilizan para que se genere el alquiler y los plazos para ejecutar los cobros.

Así mismo, (Rivera-Godoy, 2012), toma en cuenta variables de entrada y de salida; para las variables de entrada estan involucrados procesos de innovación, exploración y mejora práctica, acciones de invención en ganancia y proceso, y las actividades innovadoras en marketing y organización, para lo cual las actividades de costo y gasto se identifican de la siguiente manera:

$\mathrm{C}_{\mathrm{em}}=$ costo y gasto de exploración y mejoras prácticas

$\mathrm{C}_{\mathrm{r}}=$ costo y gasto de exploración en mejoras de renta 
Carlos Francisco López-Intriago; Juan Carlos Erazo-Álvarez; Cecilia Ivonne Narváez-Zurita; Verónica Paulina Moreno

$\mathrm{C}_{p}=$ costo y gasto de exploración en mejoras de proceso

$\mathrm{C}_{\mathrm{m}}=$ costo y gasto de exploración en mejoras de marketing

$\mathrm{C}_{0}=$ costo y gasto de exploración en mejoras organizacionales

La intensidad en la capitalización de la inversión de cada una de las actividades se expresa así:

$l_{\mathrm{em}}=$ Activos netos estratégicos determinados a exploración y mejoras prácticas

$I_{r}=$ Activos netos estratégicos determinados a invención de renta

$I_{p}=$ Activos netos estratégicos determinados a invención de proceso

Im = Activos netos estratégicos determinados a invención de marketing

$\mathrm{l}_{0}=$ Activos netos estratégicos determinados en mejoras organizacionales

Los recursos financieros necesarios para la invención cada uno de ellos tiene un costo, estos son:

$\mathrm{K}_{\mathrm{P}}=$ Costo del capital propio

$\mathrm{K}_{\mathrm{c}}=$ Costo de capital

Para el caso de las variables de salida se encuentran presentes los indicadores financieros básicos ROA, costo de capital, EVA, VMA y valor de la organización.

\section{Análisis del indicador de retorno sobre activos (ROA)}

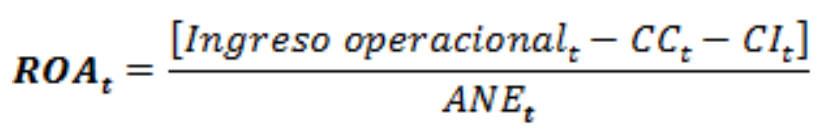

Donde:

$C C_{t}=$ Costos y gastos estratégicos originados por acciones corrientes.

$\mathrm{Cl}_{t}=$ Costos y gastos estratégicos originados por acciones innovadoras, viene expresado de la siguiente manera:

$$
\boldsymbol{C I}_{\boldsymbol{t}}=C_{\mathrm{em}}+C_{r_{t}}+C_{p_{t}}+C_{m_{t}}+C_{o_{t}}
$$

Análisis del indicador de activos netos estratégicos (ANE)

$$
A N E_{t}=I_{c_{t}}+I_{e m_{t}}+I_{r_{t}}+I_{p_{t}}+I_{m_{t}}+I_{o_{t}}
$$


Carlos Francisco López-Intriago; Juan Carlos Erazo-Álvarez; Cecilia Ivonne Narváez-Zurita; Verónica Paulina Moreno

Replanteando la expresión (1) con las expresiones (2) y (3), se obtiene:

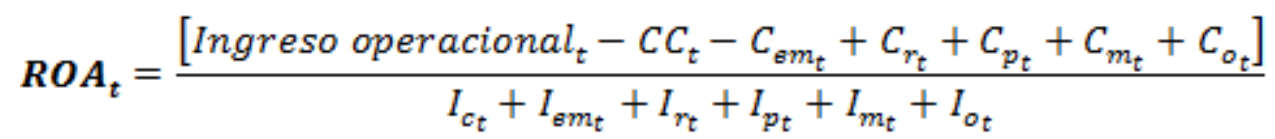

\section{Análisis del Costo de Capital $\left(\mathrm{Kc}_{\mathrm{c}}\right)$}

$K_{C_{t}}=\left(\frac{I_{C_{t}}}{A N E_{t}}\right) K_{o y_{t}}+\left[\left(\frac{A N E_{t}-I_{C_{t}}}{A N E_{t}}\right) K_{o x_{t}}\right]$

Donde:

$\mathrm{K}_{\mathrm{c}}=$ Costo del capital de los fondos aplicados a los movimientos de la organización.

$K_{\text {oy }}=$ Costo de capital para los recursos financieros aplicados a movimientos no innovadores.

Kox $=$ Costo de capital para los recursos financieros aplicados a movimientos innovadores.

\section{Valor económico agregado (EVA)}

$E V A_{t}=R O A_{t}-A N E_{t} \times K_{C_{t}}$

Valor de la organización (VO)

$$
\boldsymbol{V O}=A N E_{t-1}+V M A
$$

Extendiendo mas la expresión (7), tenemos:

$$
\boldsymbol{V O}=A N E_{t-1}+\sum_{t=1}^{t=n} \frac{\left[R O A_{t}-A N E_{t} \times K_{C_{t}}\right]}{\left(1+K_{c_{t}}\right)^{t}}
$$

Estos valores obtenidos permitiran ejecutar análisis con las muestras del sector, con los objetivos de la organización y los archivos pasados de indicadores (Lazo-Torres, ErazoÁlvarez, \& Narváez-Zurita, 2019). 
Carlos Francisco López-Intriago; Juan Carlos Erazo-Álvarez; Cecilia Ivonne Narváez-Zurita; Verónica Paulina Moreno

\section{La rentabilidad, el resultado de la creación de valor}

La rentabilidad se define como la diversificación del capital de la organización y su fortalecimiento en la busqueda de nuevos mercados y la reinversín de sus utilidades (Zamora-Torres, 2011). Un factor importante de rentabilidad en las organizaciones que se dediquen a los alojamientos y servicios se da acorde a la demanda que tengan y los valores que oferten sin afectar su economía, pues de manera general se consideran vulnerables a la competencia por desconocimiento de plazas y promociones que tengan para la captación de clientes. Además, existe una dependencia entre utilidad e inversión debido a las actividades en la organización, reflejada por los beneficios generados por el alquiler y las inversiones, su naturaleza, exactitud y credibilidad se observan en las utilidades.

En referencia a las utilidades, estas se generan en base a una planificación constante y eficiente por parte de los directivos de la organización, dando cumplimiento con los objetivos planteados para generar credibilidad ante el sector comercial; de la misma manera se cataloga como estrategia en las actividades de la organización. Los ratios financieros se obtienen al analizar el balance de rentas y gastos, estos son indicadores que proveen información y para que los directivos realicen una toma de decisiones acorde a los resultados; a traves de los cuales permite conocer la liquidez y solvencia del negocio, en caso de necesitar ayuda externa mediante créditos financieros, los banqueros pueden analizar los ratios de la organización, conociendo la situación económica del negocio durante un periodo de tiempo (Escribano-Ruiz, 2011).

Por otro lado, (Herrera-Freire, Betancourt-Gonzaga, Herrera-Freire, Vega-Rodríguez \& Vivanco-Granda, 2017), sostienen lo siguiente en cuanto a los tipos de margenes y el punto de equilibrio, utilidades; entre lo que tenemos:

1. El margen de utilidad se mide en relación a la diferencia entre inversión, ventas y gastos generados en las actividades económicas de la organización. 
Carlos Francisco López-Intriago; Juan Carlos Erazo-Álvarez; Cecilia Ivonne Narváez-Zurita; Verónica Paulina Moreno

2. El margen bruto se obtiene mediante el total de ingresos por ventas menos el costo de ventas, esto corresponde al valor de la utilidad sin considerar la resta de los gastos administrativos, de ventas y financieros.

3. El punto de equilibrio dentro del sistema financiero se refiere al valor medio de ventas, donde los costos fijos como variables se hallan numericamente iguales, es decir, el centro donde la organización no gana ni pierde.

4. La Utilidad se resta a los gastos administrativos y de ventas al valor obtenido de la utilidad bruta, posteriormente en base al valor obtenido se procede a efectuar el descuento de las utilidades a los integrantes de la organización que representa el $15 \%$ y al saldo se le resta el impuesto a la renta.

5. El cash flow o balance de flujo de efectivo permite conocer cuanto dinero posee la organización luego de restar todos los gastos concernientes a pagos realizados al giro del negocio.

Por otro lado, (Pérez-Méndez, Castro-Pérez \& García-Cornejo, 2009), sostienen que esta información es relevante al momento de realizar los indicadores financieros puesto que permite conocer en términos porcentuales los aspectos de liquidez, solvencia y rentabilidad del negocio, representando en forma general liquidez y buena reputación en la empresa en el caso de venta de acciones o al solicitar un crédito bancario.

Para (Fajardo-Ludizaca \& Álvarez-Farez, 2014), el indicador de plazas ocupadas es una óptima medida en el sector residencial generado por evaluación del servicio ofertado a los estudiantes, de esta manera, se examinan los indicadores representados por la cantidad de plazas ocupadas de manera directa pero inversamente a la cantidad de plazas ofrecidas, por 10; y al ser indicador tiene que ser un número absoluto. Esta expresado en la siguiente fórmula:

$\boldsymbol{P O}=\left(\frac{\text { Plazas Ocupadas }}{\text { Plazas Ofrecidas }}\right) \times 10$ 
Carlos Francisco López-Intriago; Juan Carlos Erazo-Álvarez; Cecilia Ivonne Narváez-Zurita; Verónica Paulina Moreno

\section{MÉTODO}

La investigación es de tipo descriptiva con un enfoque cuantitativo (Hernández, Fernández \& Baptista, 2014), el método aplicado fue el deductivo, demostrando la factibilidad económica y financiera de este aplazamiento; de esta manera, se considera como referencia un estudio de caso que se realiza a partir de la investigación en la residencia estudiantil NIA. El diseño de la investigación fue de tipo no experimental, analizando las variables involucradas: gestión financiera basada en la creación de valor. Con los resultados obtenidos se utilizó el método histórico - lógico, con el método histórico proporsiono estudiar hechos pasados, con la metodología lógica se obtuvo demostrar las causas y efectos del fenómeno encontrado. Se empleó también el método deductivo - inductivo, se desarrolla el modelo de gestión financiera que optimice la creación de valor en la Residencia Estudiantil NIA; por tanto, se enfoca directamente la planificación de actividades desarrollada acorde a la estrategia de comercialización que tiene la organización, basadas en datos operativos que expresen rendimientos financieros económicos globales y consolidados planteados inicialmente en el presupuesto operativo anual de actividades (Llivicura-Ávila, Erazo-Álvarez, \& Narváez-Zurita, 2019).

En cuanto a la recolección, tabulación y síntesis de la información se obtuvo de las encuestas e indicadores, procesada a través de la estadistica descriptiva. El método sistémico facilitó crear abstracciones de los hechos encontrados para explicar la realidad, este instrumento de trabajo es una aproximación intuitiva que se utilizó frente al objeto de investigación para una mejor comprensión. Con el método sistémico, se diseñó un modelo de gestión financiera para creación de valor aplicado a la residencia estudiantil Nia.

\section{Universo de estudio y tratamiento muestral}

El universo de estudio fue finito, es decir 22 personas que habitan en la residencia estudiantil Nia, se aplicó al 100\% de personas distribuidos de la siguiente manera: 
Carlos Francisco López-Intriago; Juan Carlos Erazo-Álvarez; Cecilia Ivonne Narváez-Zurita; Verónica Paulina Moreno

- Gerencia 1

- Administración 1

- Servicios 1

- Arrendatarios 19

\section{Tratamiento estadístico de la información}

Se obtuvieron los datos mediante cuestionarios y por medio de los formularios realizados por Google (https://docs.google.com/forms), estos fueron procesados en tablas de datos que recopilan respuestas de las personas involucradas en la investigación, se graficaron los principales resultados en el sistema Excel versión 2016, los mismos que demuestran los resultados mas importantes en el programa.

\section{RESULTADOS}

Los principales resultados de la aplicación de los instrumentos de investigación a los arrendatarios de las habitaciones y departamentos que emplean los servicios de hospedaje de la Residencia estudiantil Nia se muestran a continuación:

\section{Caracterización del agente económico}

Uno de los problemas para encontrar un arriendo es el género del arrendador, generalmente para las mujeres es mas facil, por el hecho de que el sexo masculino tiende a ser otro tipo de actividades dentro de las habitaciones como por ejemplo, consumo de bebidas alcohólicas, esto se demuestra con las respuestas obtenidas donde las arrendatarias son el $65 \%$ mientras que el género masculino de la residencia estudiantil Nia es del 35\%.

Los usuarios de mayor afluencia oxilan entre 17 a 26 años con un porcentaje del $46 \%$, quienes migran a la ciudad de Cuenca para realizar sus estudios universitarios de tercer nivel, siendo la mayoría de la costa y oriente, también existen estudiantes entre 37 a 36 
Carlos Francisco López-Intriago; Juan Carlos Erazo-Álvarez; Cecilia Ivonne Narváez-Zurita; Verónica Paulina Moreno

años quienes cursan fin de carrera de tercer nivel y estudios de cuarto nivel y personas de entre 37 a 46 años cuyo fin es realizar maestrías.

Toda clase de comunicación es importante al momento de tener un negocio, según los datos obtenidos, los usuarios de Residencia estudiantil Nia en un $62 \%$ expresaron que les recomendaron la residencia, el $31 \%$ señaló que a través de la web, obtuvo información y utilzia los servicios de la residencial, mientras que un $4 \%$ manifestó haberse enterado por medio del periódico y en el sitio $4 \%$.

Generalmente el usuario estudiantil al seleccionar un lugar para vivir busca ciertos factores de acuerdo a sus gustos y preferencias, caso particular, los arrendatarios de esta residencia estudiantil se enfocan a la infraestructura y buen trato el $5,79 \%$, las normas de convivencia el $5,60 \%$, intimidad y seguridad el $5,51 \%$, cercanía y precio, wifi y baño el 5,46\% respectivamente, servicios y ubicación el 5,37\%, cocina el 5,32\%, entretenimiento el $4,81 \%$, el $4,76 \%$ libertad para recibir a visitas, el $4,62 \%$ menicona que se fija en el género de los demás arrendatarios, el 4,39\% biblioteca, 4,30\% entretenimiento, $4,16 \%$ Tv Cable, $4,11 \%$ seguridad y $2,71 \%$ sauna.

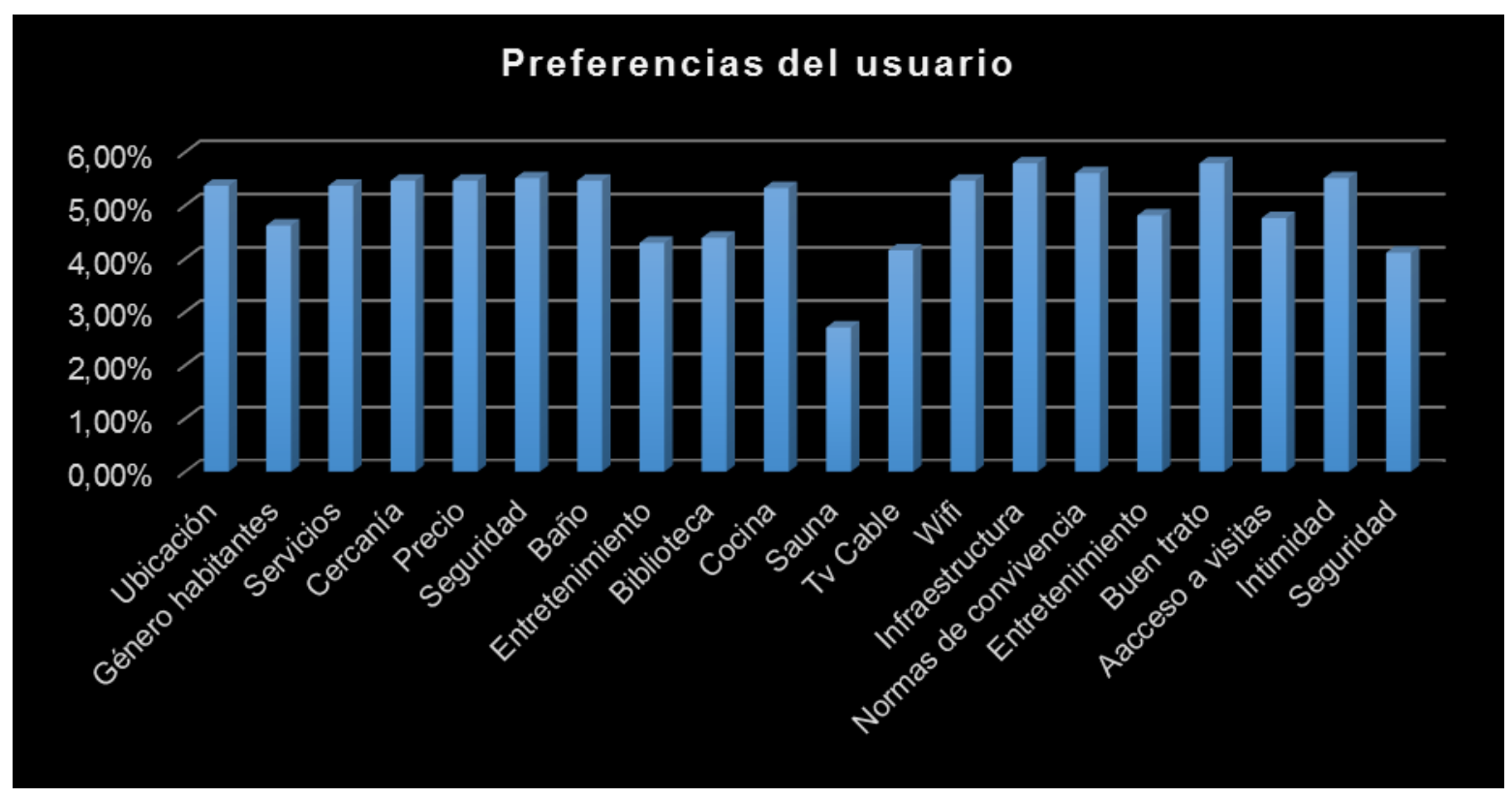

Figura 1. Preferencias del usuario arrendatario 
Carlos Francisco López-Intriago; Juan Carlos Erazo-Álvarez; Cecilia Ivonne Narváez-Zurita; Verónica Paulina Moreno

\section{PROPUESTA}

Un modelo de gestión permite administrar correctamente los recursos que dispone un negocio con el propósito de que la utilidad neta se encuentre dentro de los márgenes de rentabilidad para sus accionistas, por lo tanto, se presenta la siguiente propuesta.

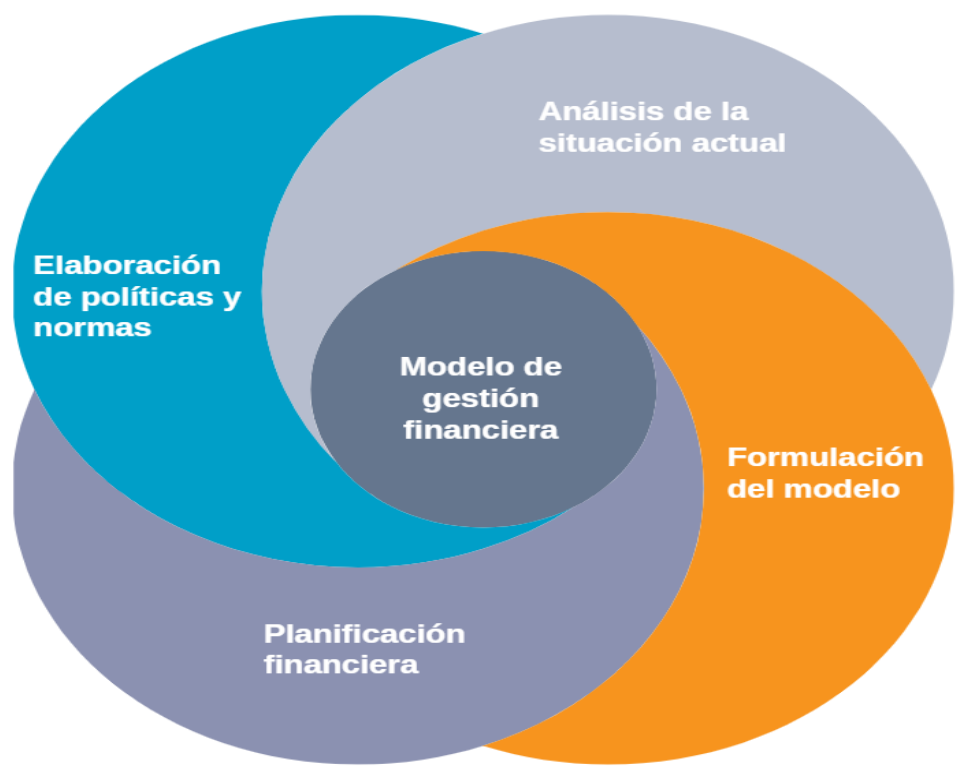

Figura 2. Esquema de la propuesta

\section{Modelo de gestión financiera}

La gestión financiera reconoce una dificultad en el manejo de las operaciones económicas de la organización; es necesario efectuar una evaluación detallada, para esto y en el primer caso se examina la situación actual del negocio en estudio.

Análisis de la situación actual de Residencia estudiantil Nia

En la tabla 2 se muestra la matriz FODA de la Residencial estudiantil Nia, estas son: 
Revista Arbitrada Interdisciplinaria KOINONIA

Año V. Vol V. №10. Julio - Diciembre 2020

Hecho el depósito de Ley: FA2016000010

ISSN: 2542-3088

FUNDACIÓN KOINONIA (F.K). Santa Ana de Coro. Venezuela.

Carlos Francisco López-Intriago; Juan Carlos Erazo-Álvarez; Cecilia Ivonne Narváez-Zurita; Verónica Paulina Moreno

\section{Tabla 2}

Matriz FODA de diagnóstico de la Residencia estudiantil Nia

\begin{tabular}{|c|c|}
\hline Debilidades & Amenazas \\
\hline Falta de estrategia socialmedia publicitaria & Baja de costos por la competencia \\
\hline Falta de seguridad privada las 24 horas & $\begin{array}{l}\text { Presencia de otras empresas en el sector de } \\
\text { ubicación de Nia }\end{array}$ \\
\hline No cuenta con un sistema financiero definido & Sector alejado de los medios de transporte \\
\hline Fortalezas & Oportunidades \\
\hline Experiencia y fiabilidad & Crear contendios de calidad \\
\hline Deferenciación de los servicios & Uso de sistema de facturación electrónica \\
\hline Contacto y seguimiento con los clientes & $\begin{array}{l}\text { Conocer las nuevas tendencias y gustos de los } \\
\text { consumidores }\end{array}$ \\
\hline
\end{tabular}

\section{Formulación del modelo}

El modelo conceptual consta de tres etapas, empezando por un paso relevante para la coordinación de operaciones y preparación financiera y presupuestaria; seguido de la guía para ejecución y revisión de los detalles financieros; y culminar en la gestión y el apoyo a la toma de decisiones.

Planificación financiera

Esta etapa representa a los estados financieros iniciales que tiene la empresa, considerando la codificación de cada cuenta y la elaboración de flujo de caja constante. Estados financieros iniciales de Residencia estudiantil Nia

En esta fase se propone estructurar el presupuesto, plan de cuentas, balances e indicadores.

\section{Presupuesto}

Costos y gastos

El presupuesto tiene como objetivo organizar la lista de cuentas que van a ser utilizadas durante un periodo, en esta sección se debe tener en cuenta la diferencia entre los costos y los gastos, los costos son aquellos rubros incurridos como parte directa del servicio como servicios básicos, alquiler del residencial (debido a que la actividad del 
Carlos Francisco López-Intriago; Juan Carlos Erazo-Álvarez; Cecilia Ivonne Narváez-Zurita; Verónica Paulina Moreno

negocio es el subarriendo) y son recuperables. Los gastos abarcan pago al personal administrativo, publicidad, servicios profesionales (contador, abogado), en la fase 2 (ejecución), se detalla las 2 cuentas principales y subcuentas relacionadas a costos y gastos. Para control interno se sugiere registrar la información en el siguiente formato.

Tarjeta de control de costos y gastos

\begin{tabular}{|c|c|c|c|c|c|}
\hline \multirow[t]{2}{*}{ Fecha } & \multirow[t]{2}{*}{ Cuenta } & \multicolumn{2}{|c|}{ Costo } & \multicolumn{2}{|c|}{ Gasto } \\
\hline & & V. Unit. & Total & V. Unit. & Total \\
\hline
\end{tabular}

Total \$

Tabla 3. Tarjeta de control de costos y gastos

Plan de cuentas en base a los requerimientos de Residencial estudiantil Nia

Tabla 4

Plan de cuentas

\section{Residencia estudiantil Nia}

Plan de cuentas

\begin{tabular}{|c|c|c|c|}
\hline 1. & Activo & & \\
\hline 1.1 & Activo corriente & 4. & Ingresos \\
\hline 1.1 .01 & Caja & 4.1 & Ingresos operacionales \\
\hline 1.1 .02 & Bancos & 4.1 .01 & Venta de servicios \\
\hline 1.1 .02 .01 & Banco Guayaquil & 4.1 .02 & Utilidad bruta en ventas \\
\hline 1.1 .03 & Cuentas por cobrar & 5. & Costos y gastos \\
\hline 1.1 .04 & $\begin{array}{l}\text { (.) Provisión cuentas } \\
\text { incobrables }\end{array}$ & 5.1 & Costos \\
\hline 1.1 .05 & $\begin{array}{l}\text { Inventario de suministros y } \\
\text { materiales }\end{array}$ & 5.1 .01 & Nómina de trabajadores \\
\hline 1.1 .06 & Crédito tributario & 5.1 .02 & Materiales y suministros \\
\hline & Activo no corriente & 5.1 .03 & Servicios básicos \\
\hline 1.2 .01 & Muebles y enseres & 5.1.03.01 & Agua \\
\hline 1.2 .02 & (.) Depreciación acumulada & 5.1 .03 .02 & Internet \\
\hline
\end{tabular}


Carlos Francisco López-Intriago; Juan Carlos Erazo-Álvarez; Cecilia Ivonne Narváez-Zurita; Verónica Paulina Moreno

\begin{tabular}{|c|c|c|c|}
\hline & muebles y enseres & & \\
\hline 2. & Pasivo & 5.1 .03 .03 & Energía eléctrica \\
\hline 2.1 & Pasivo corriente & 5.1 .03 .04 & $\begin{array}{l}\text { Depreciación de muebles y } \\
\text { enseres }\end{array}$ \\
\hline 2.1 .01 & Cuentas por pagar & 6. & Gastos \\
\hline 2.1 .02 & IESS por pagar & 6.1 & Gastos administrativos \\
\hline 2.1 .03 & Servicios básicos por pagar & 6.1 .01 & Pago de sueldos \\
\hline 3. & Patrimonio & 6.1 .02 & Aporte patronal \\
\hline 3.1 & Capital & 6.1 .03 & Cuentas incobrables \\
\hline 3.1 .01 & Aporte de capital & 6.1 .04 & $\begin{array}{l}\text { Depreciación de muebles y } \\
\text { enseres }\end{array}$ \\
\hline 3.2 & Resultado del ejercicio & 6.1 .05 & Servicios bancarios \\
\hline 3.2 .01 & $\begin{array}{l}\text { Utilidad o pérdida del } \\
\text { ejercicio }\end{array}$ & 6.2 & Cuentas transitorias \\
\hline 3.2 .02 & Utilidad neta del ejercicio & 6.2 .01 & $\begin{array}{l}\text { Resumen de pérdidas y } \\
\text { ganancias }\end{array}$ \\
\hline
\end{tabular}

A continuación, se necesita la preparación presupuestaria, en este caso se desarrolló en base a las facturas de ingresos, costos y gastos de enero de 2020.

\section{Tabla 5}

\begin{tabular}{|c|c|c|c|c|c|c|c|}
\hline Inaresos & & & & & & & \\
\hline $\begin{array}{l}\text { Alquiler de } \\
\text { dormitorios }\end{array}$ & 22 & $\$ 120,00$ & $\$ 2.640,00$ & $\begin{array}{l}\text { Costos } \\
\text { Alquiler residencial }\end{array}$ & $\begin{array}{c}\text { Cantidad } \\
1\end{array}$ & $\$ \$ 1.000,00$ & $\begin{array}{l}\text { v. Iotal } \\
\$ 1.000,00\end{array}$ \\
\hline \multirow{9}{*}{$\begin{array}{l}\text { Alquiler de } \\
\text { departamentos }\end{array}$} & 4 & $\$ 230,00$ & $\$ 920,00$ & Sueldo + beneficios & 1 & $\$ 482,46$ & $\$ 482,46$ \\
\hline & & & & $\begin{array}{l}\text { Servicios básicos } \\
\text { (agua, luz, internet) } \\
\text { Gastos }\end{array}$ & 1 & $\$ 161,36$ & $\$ 161,36$ \\
\hline & & & & Sueldo + beneficios & 1 & $\$ 568,23$ & $\$ 568,23$ \\
\hline & & & & $\begin{array}{l}\text { Servicios } \\
\text { profesionales contador }\end{array}$ & 1 & $\$ 40,00$ & $\$ 40,00$ \\
\hline & & & & $\begin{array}{l}\text { Servicios } \\
\text { profesionales abogado }\end{array}$ & 1 & $\$ 40,00$ & $\$ 40,00$ \\
\hline & & & & $\begin{array}{l}\text { Gastos suministros de } \\
\text { limpieza }\end{array}$ & 1 & $\$ 30,00$ & $\$ 30,00$ \\
\hline & & & & $\begin{array}{l}\text { Depreciación activos } \\
\text { fijos }\end{array}$ & 1 & $\$ 45,72$ & $\$ 45,72$ \\
\hline & & & & Otros gastos & 1 & $\$ 40,00$ & $\$ 40,00$ \\
\hline & & & & Gastos financieros & 1 & $\$ 16,00$ & $\$ 16,00$ \\
\hline Total & & & $\$ 3.560,00$ & Total & & & $\$ 2.423,78$ \\
\hline
\end{tabular}


Revista Arbitrada Interdisciplinaria KOINONIA

Año V. Vol V. №10. Julio - Diciembre 2020

Hecho el depósito de Ley: FA2016000010

ISSN: 2542-3088

FUNDACIÓN KOINONIA (F.K). Santa Ana de Coro. Venezuela.

Carlos Francisco López-Intriago; Juan Carlos Erazo-Álvarez; Cecilia Ivonne Narváez-Zurita; Verónica Paulina Moreno

\section{Balances financieros}

Tabla 6

Estado de Resultados

\section{Residencia estudiantil Nia \\ Balance de resultados \\ Expresado en Dólares Americanos}

\section{$4 . \quad$ Ingresos}

4.1 Ingresos operacionales

4.1.01 Ventas

5.1.05 Costo de productos vendidos

5.1.06 Variación del costo (+)

Total de ingresos

$\$ 3.560,00$

$\$ 1.628,82$

$\$ 15,00$

6. Gastos

6.1 Gastos de administración

6.1.01 Pago de sueldos

6.1.02 Aporte patronal

$\$ 568,23$

$\$ 53,13$

6.1.03 Cuentas incobrables

6.1.04 Depreciación de muebles y enseres

6.1.05 Gastos profesionales

6.1.06 Servicios bancarios

Total gastos de administración

(=) Utilidad o pérdida del ejercicio
$\$ 1,32$

$\$ 45,72$

$\$ 80,00$

$\$ 16,00$
$\$ 1.916,18$

\section{$\$ 1.151,77$}

$\$ 764,40$

Cuenca,

Contadora

Tabla 7

Estado de Situación Financiera

\begin{tabular}{|c|c|c|c|c|}
\hline \multicolumn{5}{|c|}{$\begin{array}{c}\text { Residencia estudiantil Nia } \\
\text { Balance financiero } \\
\text { Expresado en Dólares Americanos }\end{array}$} \\
\hline 1. & Activo & & & $\$ 7.175,83$ \\
\hline 1.1 & Activo corriente & & $\$ 5.532 .83$ & \\
\hline 1.1 .01 & Caja & $\$ 780,00$ & & \\
\hline 1.1 .02 & Bancos & $\$ 4.456,55$ & & \\
\hline 1.1 .05 & Inventario de suministros y materiales & $\$ 60,00$ & & \\
\hline 1.1 .06 & Crédito tributario & $\$ 236,28$ & & \\
\hline 1.2 & Activo no corriente & & $\$ 1.643,00$ & \\
\hline 1.2 .01 & Mueble y enseres & $\$ 5.325,00$ & & \\
\hline 1.2 .02 & $\begin{array}{l}\text { (-) Depreciación acumulada muebles y } \\
\text { enseres }\end{array}$ & $-\$ 3.682,00$ & & \\
\hline 2. & Pasivo & & & $\$ 541,86$ \\
\hline 2.1 & Pasivo corriente & & $\$ 541,86$ & \\
\hline
\end{tabular}


Carlos Francisco López-Intriago; Juan Carlos Erazo-Álvarez; Cecilia Ivonne Narváez-Zurita; Verónica Paulina Moreno

2.1.01 Cuentas por pagar

2.1.02 IESS por pagar

2.1.03 Servicios básicos por pagar

3. Patrimonio

3.1 Capital

3.1.01 Aporte de capital

3.2 Resultado del ejercicio

3.2.02 Utilidad neta del ejercicio

Total pasivo + Patrimonio
$\$ 257,50$

$\$ 197,80$

$\$ 86,56$

$\$ 6.633,97$

$\$ 1.151,77$

$\$ 6.633,97$

$\$ 6.633,97$

$\$ 1.151,77$

$\$ 7.175,83$

Cuenca,

Gerente

Contadora

Estos balances permiten obtener la utilidad neta de un periodo contable, además son la base para desarrollar los indicadores que miden la situación financiera del negocio, mediante los índices de rentabilidad, solvencia, liquidez, etc., los administradores pueden tomar decisiones oportunas.

\section{Indicadores financieros}

Para efectos de conocimiento y determinación de la rentabilidad, solvencia y liquidez se midió los índices, como resultados los resultados de indicadores de liquidez reflejan que por cada dólar de obligación de Residencial estudiantil Nia, posee $\$ 10,21$ para pagar y restando el valor de inventarios, pese a que se trata de una empresa de servicios no comercial sin embargo se estima que por cada dólar adeudado cuentas con $\$ 10,10$ para pagar, además refleja un capital de trabajo bueno debido a las obligaciones por pagar relativamente bajas.

Los indicadores de solvencia que se realizan permiten conocer el grado de participación de terceros para con el negocio, lo que demuestra que dicha intervención es mínima con el $0,08 \%$ Residencial estudiantil Nia, el patrimonio se encuentra comprometido con el $8 \%$, Otro punto importante de medir es la rentabilidad del negocio, para los propietarios de este negocio familiar representa el 16,05\%, es decir, la inversión en este negocio es bueno. 
Revista Arbitrada Interdisciplinaria KOINONIA

Año V. Vol V. №10. Julio - Diciembre 2020

Hecho el depósito de Ley: FA2016000010

ISSN: 2542-3088

FUNDACIÓN KOINONIA (F.K). Santa Ana de Coro. Venezuela.

Carlos Francisco López-Intriago; Juan Carlos Erazo-Álvarez; Cecilia Ivonne Narváez-Zurita; Verónica Paulina Moreno

\begin{tabular}{|c|c|c|c|c|c|}
\hline \multirow{5}{*}{$\begin{array}{l}\text { Indicadores } \\
\text { de liquidez }\end{array}$} & \multirow{5}{*}{$\begin{array}{l}\text { Mide la } \\
\text { capacidad de la } \\
\text { empresa para } \\
\text { cancelar } \\
\text { obligaciones } \\
\text { contraídas a corto } \\
\text { plazo de la } \\
\text { empresa }\end{array}$} & \multirow{2}{*}{$\begin{array}{l}\text { De liquidez } \\
\text { corriente }\end{array}$} & Activo corriente & & \multirow{2}{*}{$10.21 \%$} \\
\hline & & & Pasivo corriente & & \\
\hline & & \multirow{2}{*}{ De liquidez } & Activo corriente - Inventario & & \multirow{2}{*}{$10.10 \%$} \\
\hline & & & Pasivo corriente & & \\
\hline & & $\begin{array}{l}\text { Capital de } \\
\text { trabajo }\end{array}$ & $\begin{array}{c}\text { Activos corrientes - Pasivos } \\
\text { corrientes }\end{array}$ & $\$$ & $4,990.97$ \\
\hline \multirow{8}{*}{$\begin{array}{l}\text { Indicadores } \\
\text { de } \\
\text { solvencia }\end{array}$} & \multirow{4}{*}{$\begin{array}{l}\text { Mide en que } \\
\text { grado y forma los } \\
\text { acreedores } \\
\text { intervienen dentro } \\
\text { del } \\
\text { financiamiento a } \\
\text { corto plazo de la } \\
\text { empresa }\end{array}$} & \multirow{2}{*}{$\begin{array}{l}\text { Endeudamiento } \\
\text { del activo }\end{array}$} & Pasivo total & & \multirow{2}{*}{$0,08 \%$} \\
\hline & & & Activo total & & \\
\hline & & \multirow{2}{*}{$\begin{array}{l}\text { Endeudamiento } \\
\text { patrimonial }\end{array}$} & Pasivo total & & \multirow[b]{2}{*}{$8 \%$} \\
\hline & & & Patrimonio & & \\
\hline & \multirow{4}{*}{$\begin{array}{l}\text { Mide el nivel de } \\
\text { autonomía } \\
\text { financiera }\end{array}$} & \multirow{2}{*}{$\begin{array}{l}\text { Endeudamiento } \\
\text { del activo fijo }\end{array}$} & Patrimonio & & \multirow{2}{*}{$4.04 \%$} \\
\hline & & & Activo fijo neto & & \\
\hline & & \multirow{2}{*}{ Apalancamiento } & Activo total & & \multirow{2}{*}{$1.08 \%$} \\
\hline & & & Patrimonio & & \\
\hline \multirow{4}{*}{$\begin{array}{l}\text { Indicadores } \\
\text { de } \\
\text { rentabilidad }\end{array}$} & \multirow{4}{*}{$\begin{array}{l}\text { Medir los costos, } \\
\text { gastos y la } \\
\text { utilidad }\end{array}$} & \multirow{2}{*}{$\begin{array}{l}\text { Rentabilidad } \\
\text { neta }\end{array}$} & Utilidad neta & & \multirow{2}{*}{$16.05 \%$} \\
\hline & & & Activo total & & \\
\hline & & \multirow{2}{*}{ Margen bruto } & Venta - Costos de ventas & \multirow{2}{*}{$\$$} & \multirow{2}{*}{$1,916.18$} \\
\hline & & & Ventas & & \\
\hline
\end{tabular}

Tabla 8. Indicadores financieros 
Carlos Francisco López-Intriago; Juan Carlos Erazo-Álvarez; Cecilia Ivonne Narváez-Zurita; Verónica Paulina Moreno

\section{Fase de decisiones}

\section{Medidas}

Para crear valor a un negocio existen múltiples herramientas a ser utilizadas que generan valor y permiten orientar de forma segura su operatividad. Esta pequeña empresa familiar para agregar valor puede considerar utilizar de a poco las siguientes estrategias.

1. Ampliar el servicio de alquiler de residencia para estudiantes.

2. Además de los servicios tecnológicos ofrecer servicios de alimentación.

3. Cuando la empresa tenga varias viviendas estructuradas para el negocio lo que significa incremento de clientes y personal, será necesario el monitoreo a través de indicadores de gestión para medir la eficiencia en la atención.

\section{Elaboración de políticas y normas}

Dentro de las políticas, normas y procedimientos que esta pequeña empresa debe considerar son:

1. Negociación entre las partes, en este escenario se tratan los temas en los cuales ambas partes queden en común acuerdo, tales como: valor a pagar, tiempo de duración de contrato, fechas de corte de pago.

2. Conocimiento de las normas de convivencia. Esta parte es muy importante ya que en la residencia estudiantil habitaran personas de diferentes partes del país, convirtiendo a la residencia en un lugar de diversidad étnica.

3. Firma de contratos entre el propietario y el representante del residente estudiantil. Al estar en común acuerdo y haciendo uso del departamento legal y con conocimiento en temas de arriendos, para de esta manera cualquier duda, inquietud o sugerencia quede pactado con conocimiento de causa.

4. Pago por parte del representante del residente estudiantil por un mes de garantía por posibles daños que llegaran a darse en las instalaciones por el periodo que dure el contrato, para ello se recomienda entregar un inventario indicando el 


\section{Carlos Francisco López-Intriago; Juan Carlos Erazo-Álvarez; Cecilia Ivonne Narváez-Zurita; Verónica} Paulina Moreno

estado de la instalación y de los objetos que forman parte del departamento o habitación alquilada por el residente.

\section{CONCLUSIONES}

El modelo de gestión financiera para optimizar la creación de valor en la Residencia Estudiantil Nia con los procedimientos respectivos y el tratamiento oportuno posibilitan que los objetivos de rentabilidad se vean alcanzados, por lo tanto, para efectos de introducción al mundo financiero y de gestión en términos técnicos, en primera instancia como parámetro que agrega valor al negocio, refleja imagen, confianza al usuario es el dar a conocer el fin de la misma que se plasma a través de la misión, visión y valores empresariales.

En segunda instancia, un negocio debe tener claro sus objetivos estratégicos puesto que en base a ellos se orienta el rumbo de la empresa. Desde el punto de vista contable y financiero, es imprescindible conocer los ingresos, costos y gastos que se generan en los ciclos contables, por lo tanto, el proceso contable debe ser llevado de forma permanente, se deja los cimientos básicos para que puedan ser utilizados y al término de cada periodo contable extraer la información que sirve para medir la rentabilidad, solvencia y liquidez del negocio, indispensables para la toma de decisiones.

Esta empresa luego de utilizar estas herramientas estratégicas de gestión financiera está en condiciones de decidir y proyectarse hacia el futuro, considerando su larga trayectoria en el mercado local, puede incrementar su cadena de valor e infraestructura previa la utilización de este documento que contiene la propuesta de mejora a su gestión económica y financiera.

\section{FINANCIAMIENTO}

No monetario. 
Revista Arbitrada Interdisciplinaria KOINONIA

Año V. Vol V. №10. Julio - Diciembre 2020

Hecho el depósito de Ley: FA2016000010

ISSN: 2542-3088

FUNDACIÓN KOINONIA (F.K). Santa Ana de Coro. Venezuela.

Carlos Francisco López-Intriago; Juan Carlos Erazo-Álvarez; Cecilia Ivonne Narváez-Zurita; Verónica Paulina Moreno

\section{AGRADECIMIENTO}

Al personal de la Residencia Estudiantil NIA, de la ciudad de Cuenca, provincia del Azuay; por su gran apoyo en el desarrollo de la investigación.

\section{REFERENCIAS CONSULTADAS}

Aibar-Ortiz, M. J. (2014). Gestión financiera. [Financial management]. Recuperado de https://n9.cl/rwmc

Border, J., \& Houpt, E. J. (2017). Mercado Financiero para principiantes. [Financial Market for Beginners]. España: Babelcube Inc.

Córdoba-Padilla, M. (2012). Gestión financiera. [Financial Management]. Bogotá: Ecoe Ediciones.

Escribano-Ruiz, G. (2011). Gestión financiera. [Financial Management]. Thomson Paraninfo. Madrid:

Fajardo-Ludizaca, S, \& Alvarez Farez, L. (2014). Auditoria gerencial al estado de la situación patrimonial de las empresas del sector hotelero de la ciudad de Cuenca. [Management audit to the state focused on the patrimonial situation of the companies in Cuenca's hotel sector]. Cuenca, Azuay, Ecuador. Recuperado de https://n9.cl/2v89

García-Gonzáles, A., \& Boria-Reverter, S. (2006). Los nuevos emprendedores: Creación de empresas en el siglo XXI. [The new entrepreneurs: Creating companies in the 21st century]. Barcelona: Edicions de la Universitat de Barcelona. Recuperado de https://n9.cl/241bu

Gonzales-Urbina, P. A. (2017). Gestión de la Inversión y el Financiamiento: Herramientas para la toma de decisiones. [Investment and Financing Management: Tools for decision making]. México: IMCP (Instituto Mexicano de Contadores Públicos).

Hernández, R., Fernández, C., \& Baptista, M. (2014). Metodología de la Investigación. [Research Methodology]. Mexico DF: McGraw-Hill / Interamericana Editores, S.A. DE C.V. 
Revista Arbitrada Interdisciplinaria KOINONIA

Año V. Vol V. №10. Julio - Diciembre 2020

Hecho el depósito de Ley: FA2016000010

ISSN: 2542-3088

FUNDACIÓN KOINONIA (F.K). Santa Ana de Coro. Venezuela.

Carlos Francisco López-Intriago; Juan Carlos Erazo-Álvarez; Cecilia Ivonne Narváez-Zurita; Verónica Paulina Moreno

Herrera-Freire, A., Betancourt-Gonzaga, V., Herrera-Freire, A., Vega-Rodríguez, S., \& Vivanco-Granda, E. (2017). Razones financieras de liquidez en la gestión empresarial para toma de decisiones. [Financial reasons for liquidity in business management for decision making]. Quipukamayoc, 24(46), 153-162. https://doi.org/10.15381/quipu.v24i46.13249

Lazo-Torres, N. S., Erazo-Álvarez, J. C., \& Narváez-Zurita, C. I. (2019). El Balanced Scorecard como herramienta de control interno en el sector Manufacturero. [The Balanced Scorecard as an internal control tool in the Manufacturing sector] Revista Arbitrada Interdisciplinaria Koinonía, 4(1); 125-152. http://dx.doi.org/10.35381/r.k.v4i1.374

Llivicura-Ávila, H. P., Erazo-Álvarez, J. C., \& Narváez-Zurita, C. I. (2019). Criterios de asignación de gastos para el soporte técnico en la industria manufacturera de línea blanca. [Expense allocation criteria for technical support in the white goods manufacturing industry]. Revista Arbitrada Interdisciplinaria Koinonía, 4(1); 153179. http://dx.doi.org/10.35381/r.k.v4i1.376

Milla-Gutiérrez, A. (2011). Creación de valor para el accionista. [Creation of value for the shareholder]. Madrid: Díaz de Santos.

Murillo-Vilela, C. H., Erazo-Álvarez, J. C., Quevedo-Vázquez, J. O., \& Narváez Zurita, C. I. (2019). Plan de inversión como herramienta de la gestión del capital intelectual. [Investment plan as a tool for intellectual capital management]. Revista Arbitrada Interdisciplinaria Koinonía, 4(1); 245-273. http://dx.doi.org/10.35381/r.k.v4i1.458

Ortíz, D. (2003): Los indicadores como instrumentos para la evaluación de la gestión Pública. Una investigación empírica en el ámbito municipal. [ndicators as instruments for evaluating public management. An empirical investigation at the municipal level]. Tesis Doctoral, Universidad de Granada.

Parejo-Gámir; J, Calvo-Bernardino; A, Rodríguez-Sáiz; L, Cuervo-García; A, \& AlcaldeGutiérrez, E. (2016). Manual del sistema financiero español. [Manual of the Spanish financial system]. Recuperado de https://n9.cl/pma7

Pérez-Méndez, J. A., Castro-Pérez, O., \& García-Cornejo, B. (2009). Gestión del capital circulante y rentabilidad en pymes. [Working capital management and profitability in SMEs]. Revista de Contabilidad y Dirección, 119-140. Recuperado de https://n9.cl/bsgl

Pérez-Carballo Veiga, J. F. (2015). La gestión financiera de la empresa. [The financial management of the company]. Madrid: ESIC. 
Revista Arbitrada Interdisciplinaria KOINONIA

Año V. Vol V. №10. Julio - Diciembre 2020

Hecho el depósito de Ley: FA2016000010

ISSN: 2542-3088

FUNDACIÓN KOINONIA (F.K). Santa Ana de Coro. Venezuela.

Carlos Francisco López-Intriago; Juan Carlos Erazo-Álvarez; Cecilia Ivonne Narváez-Zurita; Verónica Paulina Moreno

Prahalad, C. K., \& Ramaswamy, V. (2004). El futuro de la competencia: Creación conjunta de valor único con los consumidores. [The Future of Competition: Creating Unique Value together with Customers]. España: Gestión 2000.

Ramírez-Solano, E. (2001). Moneda, banca y mercados financieros. [Currency, banking and financial markets]. México: Pearson Educación.

Rivera-Godoy, J. A. (2012). La incidencia de la innovación sobre la creación de valor: propuesta de un modelo desde la perspectiva financiera. [The impact of innovation on value creation: Proposal for a model from the financial perspective]. Revista Facultad De Ciencias Económicas, 20(2), 175-187. https://doi.org/10.18359/rfce.2171

Rojo Ramírez, A. A. (2007). Valoración de empresas y gestión basada en valor. [Valuation of companies and value-based management]. España: Espain.

Santiago-Chávez, N. I., \& Gamboa-Salinas, J. M. (2017). Gestión financiera empresarial. [Corporate financial management]. Ambato: UTA. http://dx.doi.org/10.31164/ceuta9789978978139

Terrazas-Pastor, R. A. (2009). Modelo de gestión financiera para una organización. [Financial management model for an organization]. Perspectivas, 23; 55-72. Recuperado de https://n9.cl/0iov

Vargas-Sánchez, G. (2006). Introducción a la teoría económica. [Introduction to economic theory]. México, México: Pearson. Recuperado de https://n9.cl/pl2bd

Zamora-Torres, A. I. (2011). Rentabilidad y Ventaja Comparativa: Un análisis de los sistemas de producción de Guayaba en el estado de Michoacán. [Profitability and Comparative Advantage: An Analysis of Guava Production Systems in Michoacán State]. Recuperado de https://n9.cl/ho56

(C2020 por los autores. Este artículo es de acceso abierto y distribuido según los términos y condiciones de la licencia Creative Commons Atribución-NoComercial-Compartirlgual 4.0 Internacional (CC BY-NC-SA 4.0)

(https://creativecommons.org/licenses/by-nc-sa/4.0/). 